\title{
The ITER EC H\&CD Upper Launcher: Seismic Analysis
}

\author{
G. Aiello ${ }^{a}, A$. Vaccaro $^{a}$, D. Combescure ${ }^{b}$, R. Gessner ${ }^{\mathrm{a}}$, G. Grossettia,
}

A. Meier ${ }^{a}$, G. Saibene ${ }^{b}$, T. Scherer ${ }^{a}$, S. Schreck ${ }^{a}$, P. Spaeh ${ }^{a}$, D. Strauss ${ }^{a}$

aKarlsruhe Institute of Technology, Institute for Applied Materials, P.O. Box 3640, D-76021 Karlsruhe, Germany

${ }^{\mathrm{b}}$ Fusion for Energy, C/Josep Pla 2, Torres Diagonal Litoral-B3, E-08019 Barcelona, Spain

\section{Introduction and background}

The electron cyclotron heating and current drive (EC H\&CD) upper launcher (UL) is a component of the ITER tokamak machine devoted to inject localized high microwave power into the plasma for control of the MHD instabilities. The UL consists of an assembly of ex-vessel waveguides (with diamond windows and isolation valves) and an in-vessel port plug (PP). The UL is part of the first confinement system and so it has the most stringent requirements in the ITER safety, quality, vacuum, seismic and tritium classifications. In the event of an earthquake, the structural stability and the confinement function of the UL has to be maintained. In this work, the resistance of the UL PP to the seismic events was checked by means of FEM analyses in ANSYS Workbench. The seismic analysis of the PP was performed using the response spectrum (RS) approach and the acceleration floor response spectra (FRS) for the so-called SL-2 seismic event were applied as input. SL-2 event is the reference earthquake in ITER and is classified as a Category IV event, i.e. extremely unlikely loading condition. The damage limit for the UL associated to this category event is the faulted condition. The UL PP is mounted as a cantilever into the upper port of the vacuum vessel by a support flange at its rear side. It has a length of about $6 \mathrm{~m}$ and the gap between it and the port is $25 \mathrm{~mm}$, of which $13 \mathrm{~mm}$ only are allowed for the PP deflection. The seismic analysis of the UL PP was first run and the results are given in terms of displacements and stresses. Then, to take account of the port a second analysis of the PP integrated in the port was carried out. Finally, in this work the feasibility of a seismic analysis of the UL PP using a static approach was also investigated.

\section{Methods}

\section{Response spectrum approach}

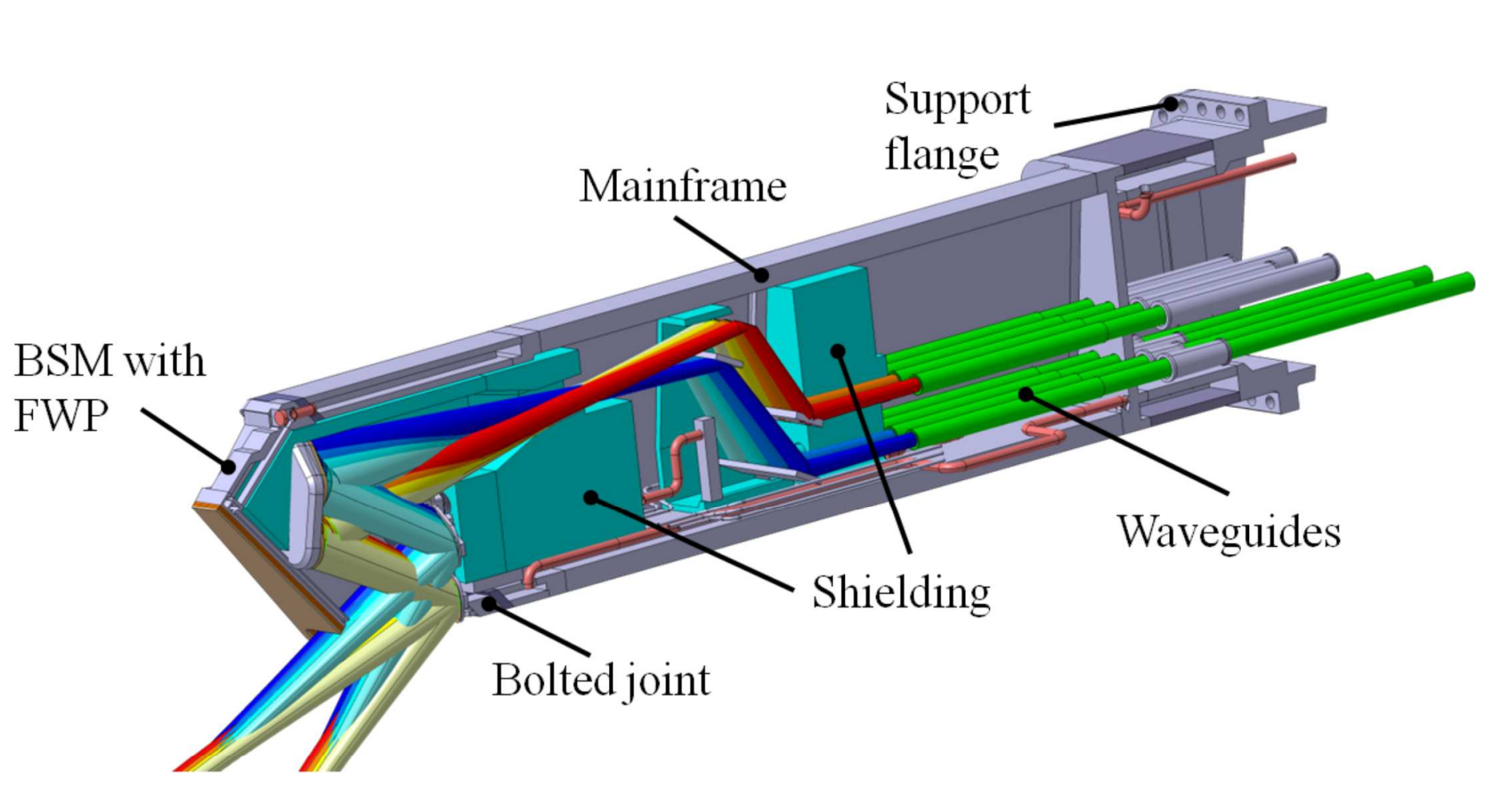

Modal analysis

- 3 RS analyses for 3 excitation directions Only the first 2 natural frequencies fall in the frequency range of the applied FRS - Missing mass correction method used for contribution of modes higher than $f_{\text {ZPA }}$ SRSS rule for modal combination

- Newmark's rule for spatial combination
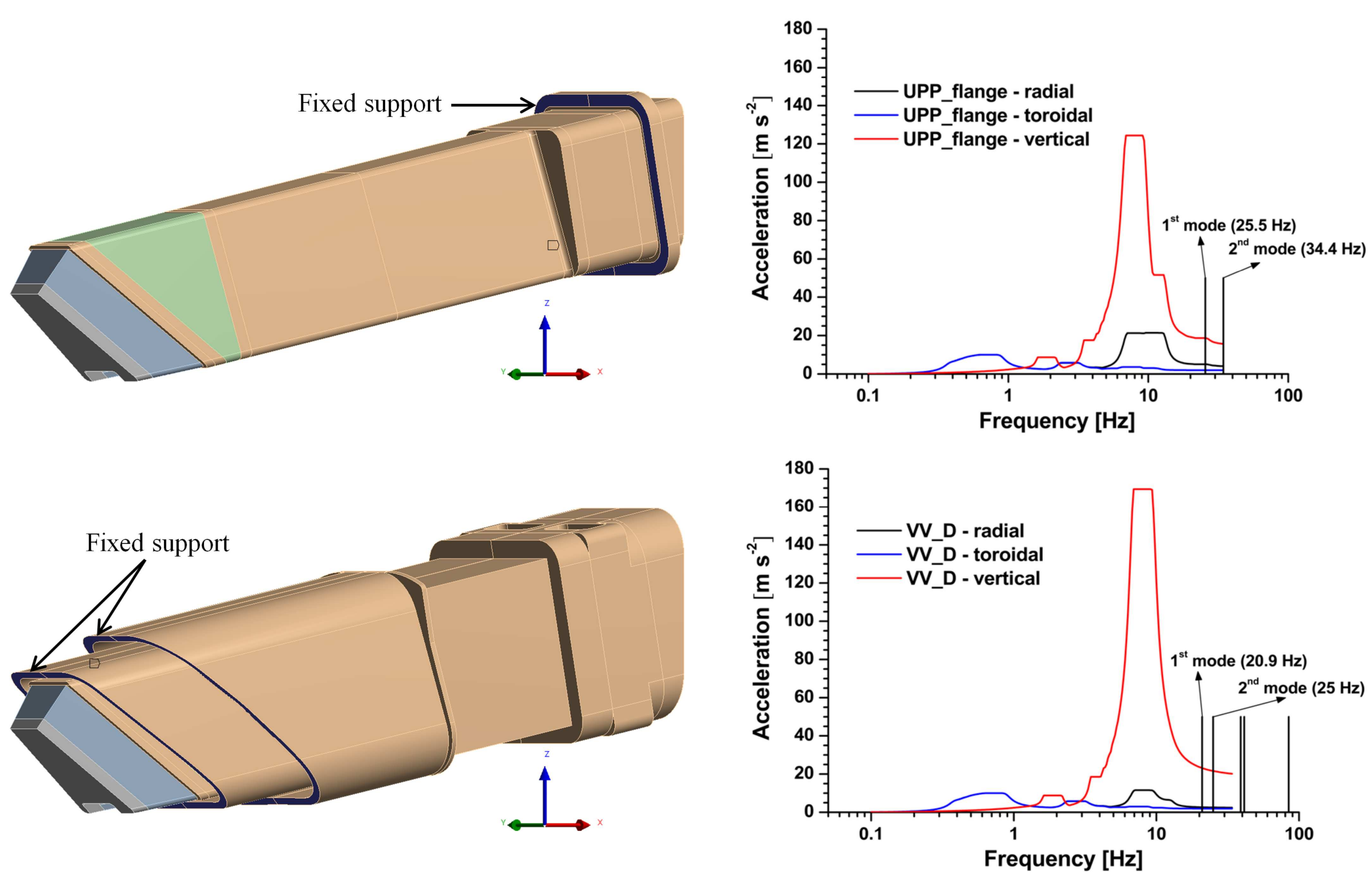

\section{Static approach}

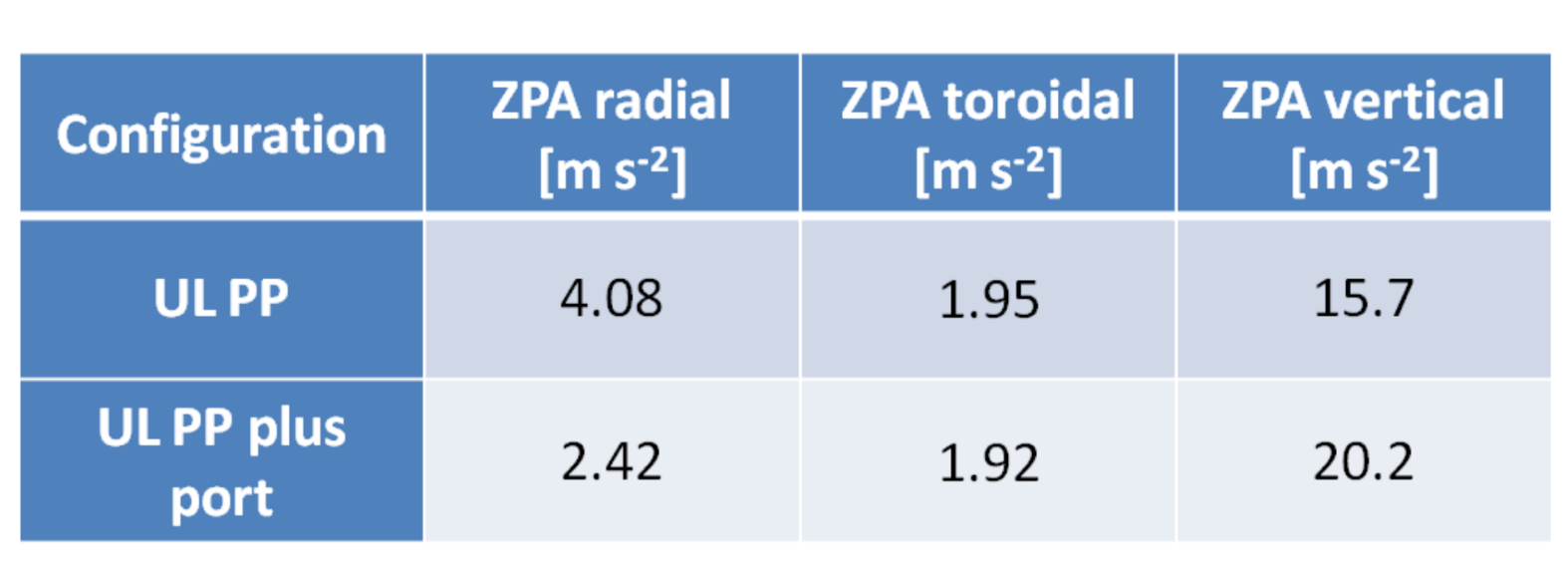

- 8 structural analyses were run for each geometrical configuration (ZPA have to be considered with plus and minus sign variations) The results from the most severe load combination were compared with the ones obtained from the RS approach
Results

Response spectrum approach

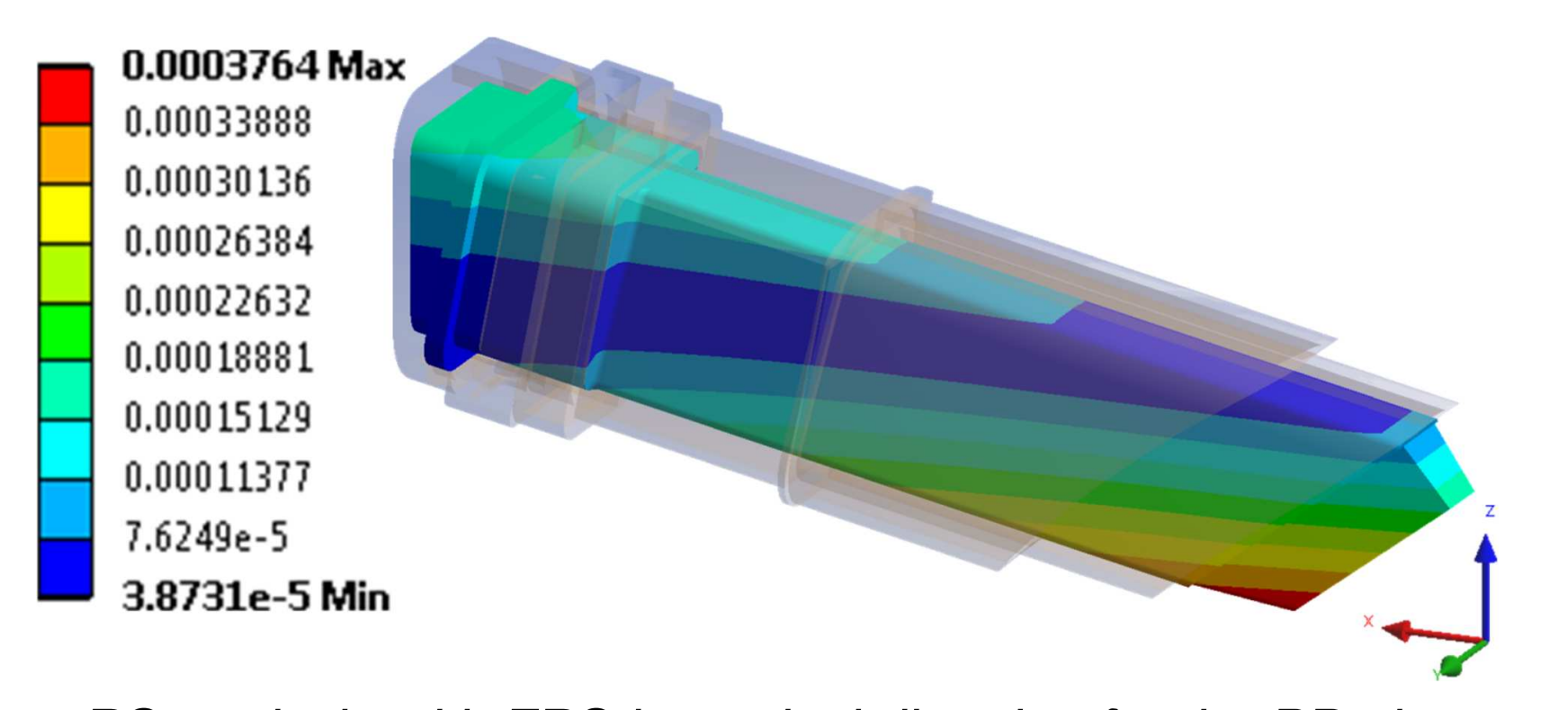

$R S$ analysis with FRS in vertical direction for the PP plus port configuration: radial displacement [m].

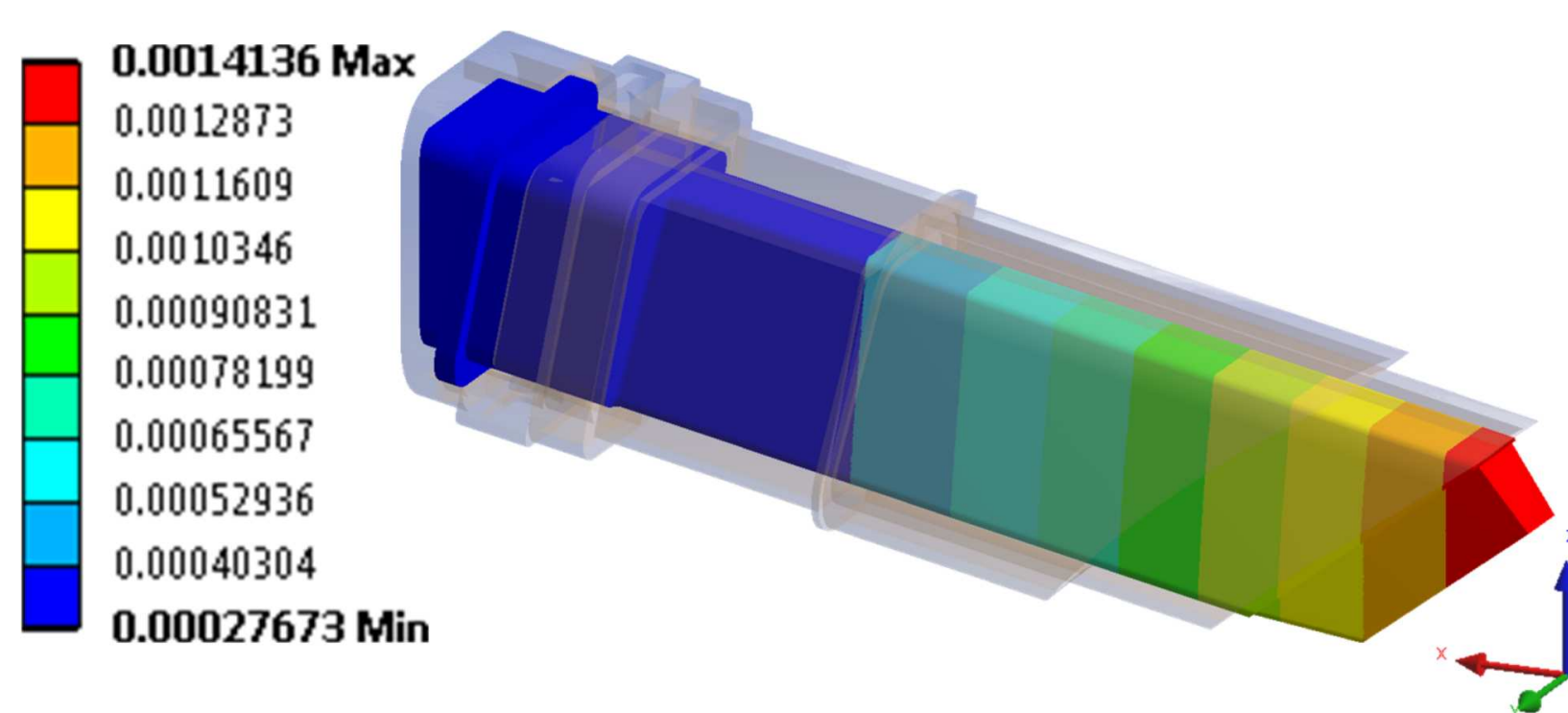
RS analysis with FRS in vertical direction for the PP plus
port configuration: vertical displacement [m].

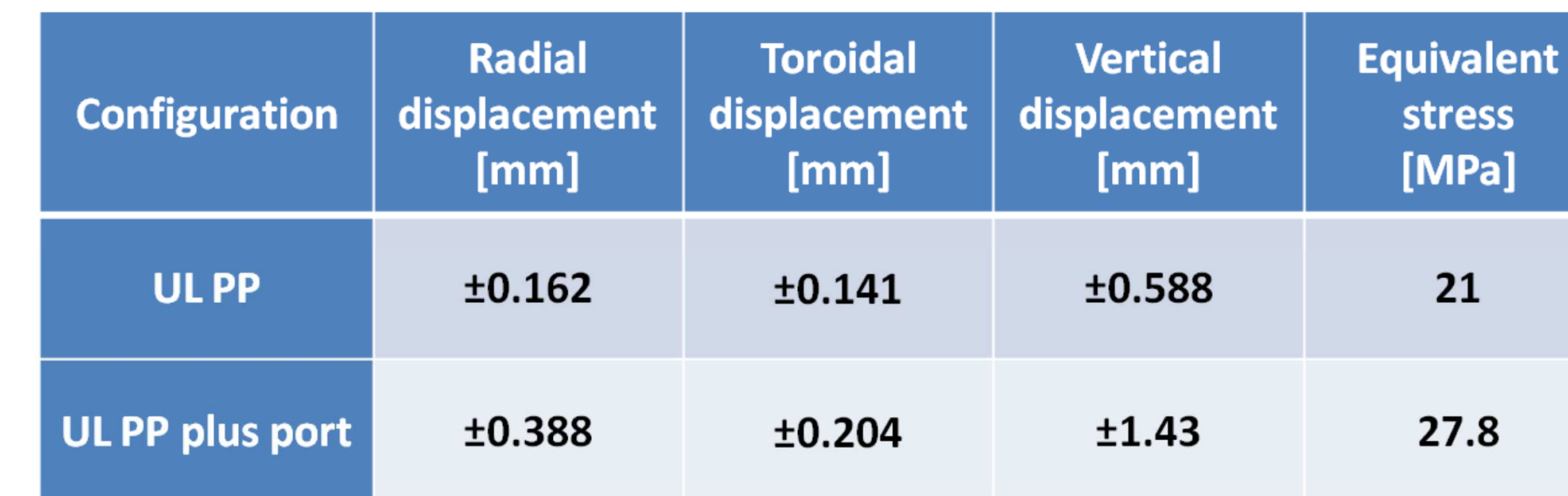

port configuration: equivalent stress $[P a]$

Maximum results obtained in the UL PP by applying Newmark's rule:

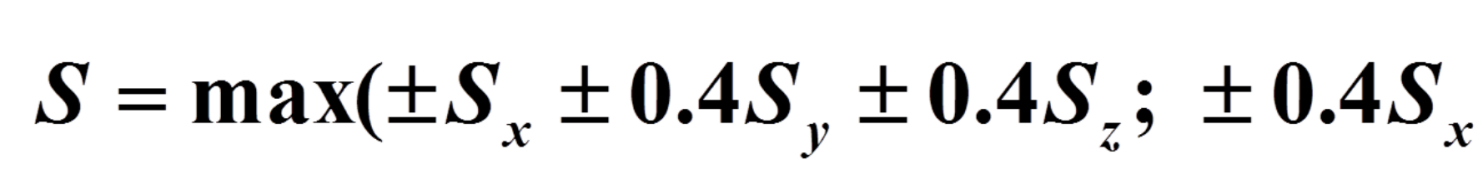

$\left.\pm S_{y} \pm 0.4 S_{z} ; \pm 0.4 S_{x} \pm 0.4 S_{y} \pm S_{z}\right)$

\section{Static approach}
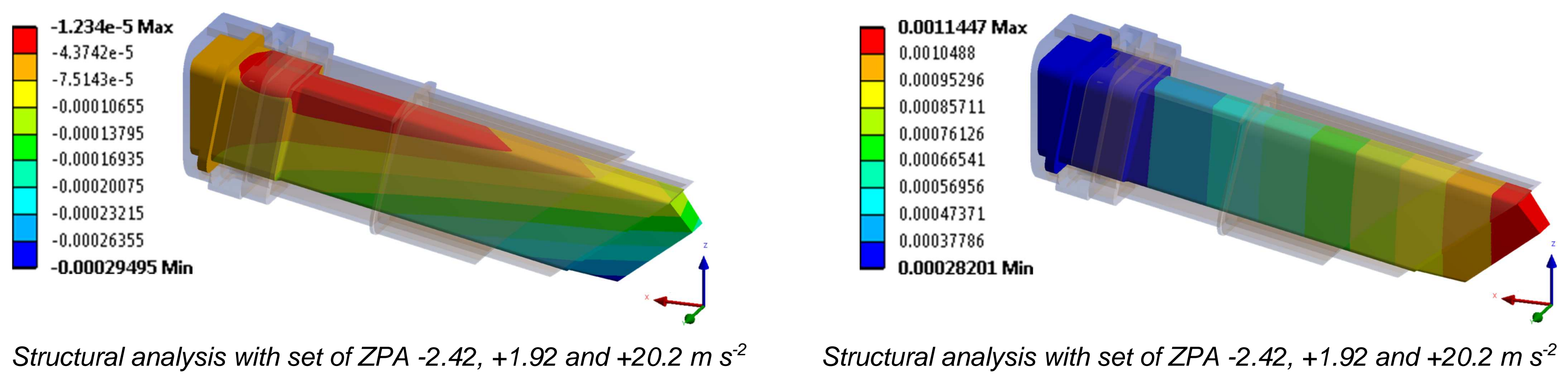

for the PP plus port configuration: radial displacement [m].

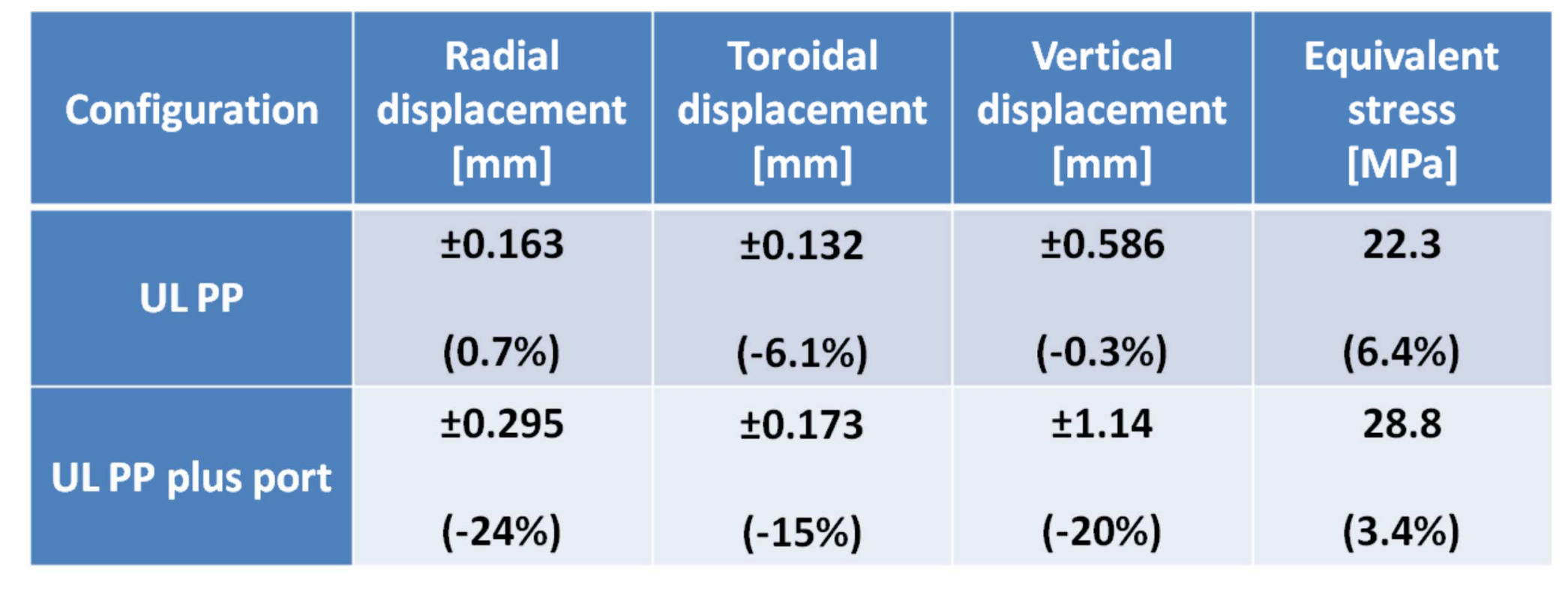

The percentages into brackets are the relative differences with respect to the correspondent results given by the RS approach.

\section{Conclusions}

- The natural frequencies of the two geometrical configurations are far from the frequencies of the FRS peaks, so no resonance condition occurs for the UL PP.

- The maximum equivalent stress is lower than $30 \mathrm{MPa}$. Due to the very high vertical excitation, the PP oscillates mainly in the vertical direction with amplitude anyway less than $2 \mathrm{~mm}$.

- The displacements obtained for the PP plus port configuration are de-facto relative displacements between $\mathrm{PP}$ and port and they can be compared with the size of the gap between the UL PP and the port.

- When the port is included in the analysis, the static approach cannot be used anymore because more natural frequencies fall in the FRS range. The RS approach has to be used for the PP plus port configuration and for homogeneity reasons it is also extended to the PP configuration.

\section{Acknowledgments}

This work was supported by Fusion for Energy under the grant contract No. F4E-2010GRT-161. The views and opinions expressed herein reflect only the author's views. Fusion for Energy is not liable for any use that may be made of the information contained therein. 